\title{
MINIMAX AND VARIATIONAL INEQUALITIES FOR COMPACT SPACES
}

\author{
J. F. MCCLENDON
}

\begin{abstract}
The minimax inequality $\min , \sup _{1} f(x, y) \leqslant \sup _{1} f(x, x)$, proved by $\mathrm{K}$ Fan for convex spaces, is proved here for certain contractible and acyclic spaces. Some variational inequality and fixed point theorems are deduced.
\end{abstract}

In [10] Fan proved a minimax inequality for $f: X \times X \rightarrow R$, namely,

$$
\min _{y} \sup _{x} f(x, y) \leqslant \sup _{x} f(x, x) .
$$

Fan assumed $X$ was a compact convex subset of a Hausdorff topological vector space $E$ and, also, made some assumptions about $f$. In the present paper the inequality is proved under different assumptions on $X$ and $f$. The main generalization is the weakening of "convex" to "contractible" or "acyclic". Because of other changes neither of the minimax inequality results (\$2) strictly generalizes Fan's result. Theorem 2.1 does include Fan's result if the topological vector space $E$ is finite dimensional. In the infinite-dimensional case, Theorem 2.2 includes and generalizes compact convex subsets of a locally convex topological vector space, but imposes more restrictions on $f$. In $\$ 3$ some variational inequality (see, for example, Browder [6], Brezis-Nirenberg-Stampacchia [4], Dugundji-Granas [8] for convex analogues) and fixed point theorems are deduced from the minimax inequality.

1. Terminology, background results. The real numbers are $R$. A space is contractible if the identity map is homotopic to a constant (so a contractible space is always nonempty). A nonempty convex set is contractible-but, of course, there are many contractible sets in a topological vector space which are not convex. A nonempty space is acyclic if it is connected and its Čech homology (coefficients in a fixed field) is zero in dimensions greater than zero. Every contractible space is acyclic and there are examples showing the converse is not true (e.g., Brown [7, p. 31]).

A set valued function $m: X \rightarrow Y$ with $m(x)$ nonempty for all $x$ is called a multifunction. The graph $G(m)$ is $\{(x, y) \in X \times Y \mid y \in m(x)\} . m$ is said to be an open-graph [closed-graph] multifunction if $G(m)$ is open [closed] in $X \times Y$. If $m$ : $X \rightarrow X$ then $x \in X$ is a fixed point of $m$ if $x \in m(x)$. We will use two fixed point results: Proposition 2.3 of $\S 2$ and the following result of Begle.

1.1. Proposition (Begle [2]). Suppose $X$ is a compact acyclic lc space and $m$ : $X \rightarrow X$ is a closed-graph acyclic valued multifunction. Then $m$ has a fixed point. 
1.2. Proposition. (a) (Lefschetz) An ANR is an lc space. (b) ( R. Thompson [14]) A finite union of compact convex subsets of a locally convex topological vector space is an lc space.

See $[2,14]$ for the definition of "lc space". It will be convenient to define an $f c$ space to be a finite union of compact convex subsets of a locally convex topological vector space. The following lemma is needed. Let $f: X \rightarrow R$ be an upper semicontinuous function and let

$$
V(t)=\{x \in X \mid f(x)>t\} \quad \text { and } H(t)=\{x \in X \mid f(x) \geqslant t\} .
$$

1.3. Lemma. If $X$ is compact and $V(t)$ is acyclic for all $t$ then $H(t)$ is acyclic for all $t$.

Proof. $H(t)=\bigcap\{V(t-\varepsilon) \mid \varepsilon>0\}$. Using $X$ compact and $f$ usc, it is easily checked that $H(t) \subset$ open $W$ implies $H(t) \subset V(t-\varepsilon) \subset W$, some $\varepsilon>0$. Now the result follows from the continuity property of Čech homology.

ANR will always mean ANR (metric). The reader may find it helpful to remember that if $X$ is a subset of Euclidean space then $X$ is a compact finite-dimensional ANR iff $X$ is compact and locally contractible (see Borsuk [3, p. 122]).

Henceforth $X$ will always be a nonempty space.

2. Minimax inequality results. In this section we consider $f: X \times X \rightarrow R$, with $\sigma=\sup \{f(x, x) \mid x \in X\}$. The following two theorems will be proved.

2.1. TheOREM. Suppose that $X$ is a compact acyclic finite-dimensional ANR. Suppose also that:

(1) $\{(x, y) \in X \times X \mid f(x, y)>\sigma\}$ is open.

(2) $\{x \in X \mid f(x, y)>\sigma\}$ is contractible or empty for all $y \in X$.

Then there is a $y_{0} \in X$ with $f\left(x, y_{0}\right) \leqslant \sigma$, all $x \in X$.

The next theorem relaxes the hypotheses on $X$ but strengthens those on $f$.

2.2. THEOREM. Suppose that $X$ is compact, acyclic, and either an $f c$ space or an ANR. Suppose also

(1) $\{(x, y) \mid f(x, y)<t\}$ open, all $t \in R$,

(2) $\{y \mid f(x, y)>t\}$ open, all $x \in X$, all $t \in R$,

(3) $\{x \mid f(x, y)>t\}$ acyclic or empty, all $y \in Y$, all $t \in R$.

Then $\min _{y} \sup _{x} f(x, y) \leqslant \sigma$.

Note that if $f$ is lower semicontinuous then 2.1(1) is satisfied and, also, the conclusion of 2.1 is then equivalent to: $\min _{y} \sup _{x} f(x, y) \leqslant \sigma$. As stated, $2.2(1)$ says $f$ is upper semicontinuous and 2.2(2) says $f(x,-)$ is lower semicontinuous. However, the proof of 2.2 will show that it suffices to take $t=\sigma+\varepsilon$ for $\varepsilon$ small and positive. It will also show that $>$ can be replaced by $\geqslant$ in (3). As noted in the introduction, Theorem 2.1 includes the finite-dimensional version of Fan's result [10].

Proof of 2.1. Define $m: X \rightarrow X$ by $m(y)=\{x \mid f(x, y)>\sigma\}$. Hypothesis (1) shows that $m$ is an open-graph multifunction. The following fixed point theorem will be needed. 
2.3. Proposition [11, Corollary 3.3]. Suppose $X$ is a compact acyclic finite-dimensional $A N R$ and $m: X \rightarrow X$ a subopen multifunction with infinitely connected values. Then $m$ has a fixed point.

By hypothesis (2), $m$ has contractible so infinitely connected values. We have "subopen" is a generalization of open-graph. If $m$ is a multifunction, then the above proposition will yield a fixed point for $m$. Then there is a point $y^{\prime}$ with $y^{\prime} \in m\left(y^{\prime}\right)$. But this says that $f\left(y^{\prime}, y^{\prime}\right)>\sigma=\sup _{x} f(x, x)$ which is not possible. Hence $m$ is not a multifunction and there is a point $y_{0}$ so that $m\left(y_{0}\right)$ is empty, i.e., $f\left(x, y_{0}\right) \leqslant \sigma$ for all $x \in X$, proving 2.1 .

In [13] it is proved that "subopen" can be replaced by (for example) " $r$-open graph" in 2.3 above. Thus in 2.1 , if $L=\{(x, y) \mid f(x, y)>\sigma\}$ hypothesis (1) can be replaced by the following: (1') There is an open set $U$ of $X \times X$ and a continuous retraction $r=\left(r_{1}, r_{2}\right): U \rightarrow L$ such that $r_{2}(a, b)=b$ for all $b \in X$.

Proof of 2.2. We can assume $\sigma<\infty$ and define for each $\varepsilon>0, m(y)=\{x \mid$ $f(x, y) \geqslant \sigma+\varepsilon\}$. By hypothesis (1) $m$ is a closed-graph multifunction and (2) says that $f(x,-)$ is lower semicontinuous. (3) and Lemma 1.3 show that $m$ has acyclic values. Since $m$ cannot have a fixed point, Propositions 1.1 and 1.2 show that $m\left(y^{\prime}\right)=\varnothing$ for some $y^{\prime}=y^{\prime}(\varepsilon)$. By taking $\varepsilon=1 / n$ we get a sequence and then a convergent subnet ( $X$ compact) $y(k) \rightarrow \bar{y}$ with $f(x, y(k)) \leqslant \sigma+\varepsilon(k), \varepsilon(k) \rightarrow 0$. However, $f(x,-)$ is lower semicontinuous so $f(x, \bar{y}) \leqslant \sigma$. Thus $\sup _{x} f(x, \bar{y}) \leqslant \sigma$ and $\inf _{y} \sup _{x} f(x, y) \leqslant \sigma$ and because $X$ is compact and $f(x,-)$ is lower semicontinuous "inf" can be replaced by " min".

3. Variational inequality and fixed point theorems. We first deduce two preliminary results, along the lines of Fan [10, Corollary 1].

3.1. THEOREM. Suppose that $X$ is a compact acyclic finite-dimensional ANR. Suppose $g: X \times X \rightarrow R$ is a function such that $\{(x, y) \mid g(y, y)>g(x, y)\}$ is open and $\{x \mid g(y, y)>g(x, y)\}$ is contractible or empty for all $y \in X$. Then there is a $y_{0} \in X$ with $g\left(y_{0}, y_{0}\right) \leqslant g\left(x, y_{0}\right)$ for all $x \in X$.

Proof. Define $f: X \times X \rightarrow R, f(x, y)=g(y, y)-g(x, y)$. Then $\sigma=$ $\sup _{x} f(x, x)=0$ so $f(x, y)>\sigma$ iff $g(y, y)>g(x, y)$ and hypotheses (1) and (2) of Theorem 1.2 are satisfied. Thus there is a $y_{0} \in X$ with $f\left(x, y_{0}\right) \leqslant 0$, all $x \in X$, proving 3.1.

3.2. TheOREM. Suppose that $X$ is compact, acyclic, and either an fc space or an ANR. Let $g: X \times X \rightarrow R$ be a function such that (a) $g$ is conitinuous on the diagonal $(=\{(x, x) \mid x \in X\}),(b) g$ is lower semicontinuous, and (c) $g(x,-)$ is upper semicontinuous for all $x \in X$. Suppose also that $\{x \mid g(x, y)<s\}$ is acyclic or empty for all $s \in R$, all $y \in X$. Then there is a $y_{0} \in X$ with $g\left(y_{0}, y_{0}\right) \leqslant g\left(x, y_{0}\right)$, all $x \in X$.

Proof. Define $f: X \times X \rightarrow R, f(x, y)=g(y, y)-g(x, y)$. The hypotheses on $g$ imply that $f$ is upper semicontinuous and that $f(x,-)$ is lower semicontinuous. Thus (1) and (2) of Theorem 1.2 are satisfied. For a given $y$ and $t$ let $s=g(y, y)-t$. Then (3) of 2.1 follows from the above hypotheses on $g$. The conclusion of 3.2 then follows from that of 2.1 . 
There are a large number of variational inequality results in the literature using convexity. See, for example, Browder [5, 6], Brezis-Nirenberg-Stampacchia, [4], Allen [1], Dugundji-Granas [8]. Here we state some results for acyclic spaces.

3.3. Corollary. Let $E$ be a topological vector space with algebraic dual $E^{*}$. Suppose $E \supset X$ and $X$ is a compact acyclic finite-dimensional ANR. Suppose $T$ : $X \rightarrow E^{*}$ is a function such that

(1) $\{(x, y) \in X \times X \mid(T y, x)<(T y, y)\}$ is open,

(2) $\{x \in X \mid(T y, x)<(T y, y)\}$ is contractible or empty, all $y \in X$. Then there is a $y_{0} \in X$ with $\left(T y_{0}, y_{0}-x\right) \leqslant 0$, all $x \in X$.

3.4. Corollary. Let $E$ be a topological vector space with algebraic dual $E^{*}$. Suppose $E \supset X$ and $X$ is compact, acyclic, and either fc or ANR. Suppose $T: X \rightarrow E^{*}$ is a function such that $y \rightarrow(T y, y)$ is continuous, $y \rightarrow(T y, x)$ is upper semicontinuous, all $x$, and $(x, y) \rightarrow(T y, x)$ is lower semicontinuous. Suppose $\{x \in X \mid(T y, x)<s\}$ is acyclic or empty, all $s \in R$, all $y \in X$. Then there is a $y_{0} \in X$ with $\left(T y_{0}, y_{0}-x\right) \leqslant 0$ for all $x \in X$.

3.3 and 3.4 are proved by using $g(x, y)=(T y, x)$ in 3.1 and 3.2. A version of 3.3 that allows $X$ to be noncompact (but still finite dimensional) will be proved after the fixed point theorems below. These fixed point theorems treat a function $f: X \rightarrow M$ where $X \subset M$ and involve a condition on the "boundary". There are many theorems of this general type in the literature for convex $X$ (see, e.g., Browder $[5,6]$ ).

3.5. Definition. Suppose $X$ a subset of a metric space $(M, d)$. Define $\delta X=\delta_{M} X$ $=\{y \in X \mid$ There is a $z \in M \backslash X$ with $d(z, y)=d(z, X)\}$.

3.6. Theorem. Suppose $(M, d)$ is a metric space, $M \supset X$ and $X$ a compact, acvelic, finite-dimensional ANR. Suppose $f: X \rightarrow M$ is a continuous function with $f(\delta X) \subset X$ and $\{x \in X \mid d(y, f(y))>d(x, f(y))\}$ contractible or empty for all $y \in X$. Then $f$ has a fixed point.

Proof. Define $g: X \times X \rightarrow R$ by $g(x, y)=d(x, f y)$. Then 3.1 gives $y_{0} \in X$ with $d\left(y_{0}, f y_{0}\right) \leqslant d\left(x, f y_{0}\right)$ for all $x \in X$. Suppose, by way of contradiction, that $f\left(y_{0}\right) \in$ $M \backslash X$. Use $z=f\left(y_{0}\right)$ in the definition of $\delta X$ and conclude $y_{0} \in \delta X$. But by hypothesis, $f(\delta X) \subset X$. Thus we must have $f\left(y_{0}\right) \in X$. Now use $x=f\left(y_{0}\right)$ on the right-hand side of the above inequality and conclude $d\left(y_{0}, f y_{0}\right)=0$. So $y_{0}$ is the desired fixed point.

3.7. Theorem. Suppose $(M, d)$ a metric space, $X$ a compact acyclic lc subspace of $M$. Suppose $f: X \rightarrow M$ is a continuous function such that $\{x \in X \mid d(x, f y) \leqslant t\}$ is acyclic or empty for all $y \in X$ and $t \in R$. Then there is a $y_{0} \in X$ with $d\left(f y_{0}, y_{0}\right)=$ $d\left(f y_{0}, X\right)$.

The proof of 3.7 is just the first part of that of 3.6 but using 3.2. Note that 3.7 generalizes Theorem 2 of Fan [9].

3.8. Corollary. Suppose $M, X, f$ as in 3.7 and $f(\delta X) \subset X$. Then $f$ has a fixed point. 
Proof. Suppose, by way of contradiction, the $y_{0}$ of 3.7 is in $M \backslash X$. Then using $z=f y_{0}$ in 3.5 we see that $y_{0}$ is in $\delta X$ showing $f y_{0} \in X$. Now for $f y_{0} \in X$ the conclusion of 3.7 gives $d\left(f y_{0}, y_{0}\right)=0$ so $y_{0}$ is the desired fixed point.

We conclude with another variational inequality result. The condition $(\star)$ of the result seems a littel severe (cf. the weaker condition in Brezis-Nirenberg-Stampacchia [4]) but the methods of proof used here seem to require it.

3.9. Definition. Let $S$ be a subset of a metric space $M$. Say $S$ is simple if $S \cap B(z ; \varepsilon)$ is contractible or empty for all $z \in M$, all $\varepsilon>0$.

3.10. Theorem. Let $E$ be a topological vector space and $X$ any metric subset. Suppose $T: X \rightarrow E^{*}$ is a function such that $\{(x, y) \in X \times X \mid(T y, x)<(T y, y)\}$ is open and $\{x \in X \mid(T y, x)<(T y, y)\}$ is contractible or empty for all $y \in X$. Suppose there is a simple $K \subset X$ that is an acyclic compact finite-dimensional ANR satisfying

$$
x \in X \backslash K \Rightarrow(T y, y-x) \leqslant 0, \text { for all } y \in \delta_{X}(K) .
$$

Then there is $a y_{0} \in X$ with $\left(T y_{0}, y_{0}-x\right) \leqslant 0$, all $x \in X$.

Proof. Define $m: K \rightarrow X$ by $m(y)=\{x \in X \mid(T y, x)<(T y, y)\}$. By hypothesis $m$ is open-graph and has contractible or empty values. Assume, by way of contradiction, that $m$ has nonempty values. Then, by [12, Corollary 2.2], $m$ has a continuous selection $f: K \rightarrow X$. Let $y \in \delta K$, then $f y \in m y$ so $(T y, f y)<(T y, y)$. Then condition ( $\star$ ) with $x=f y$ shows we must have $f y \in K$. Thus $f(\delta K) \subset K$. Also $\{x \in K \mid$ $d(x, f y)<d(y, f y)\}$ is $B(f y ; \varepsilon) \cap K$ where $\varepsilon=d(y, f y)$ and this is contractible or empty since $K$ is simple. The hypotheses of 3.6 are satisfied, giving a $y^{\prime}=f y^{\prime} \in m y^{\prime}$. But this is not possible-so $m y_{0}$ must be empty for some $y_{0} \in K$. Thus $\left(T y_{0}, y_{0}\right) \leqslant$ $\left(T_{v_{0}}, x\right)$ for all $x \in X$, proving 3.10 .

\section{REFERENCES}

I. (i. Allen. Iariational incequalities, complementarity problems, and duality theorems, J. Math. Anal. Appl. 58 (1977), 1-10.

2. E. Begle, A fived poimt theorem. Ann. of Math. (2) 51 (1950), 544-550.

3. K. Borsuk, Theory of retracts, PWN, Warsaw, 1967.

4. H. Brezis, L. Nirenberg and (;. Stampacchia, A remark on Ky Fan's minimax principle, Boll. Un. Math. Ital. 6 (1972), 293-300.

5. F. Browder, $A$ new generalization of the Schauder fixed point theorem, Math. Ann. 174 (1967), $285-290$.

6. The fixed point theory of multi-valued mappings in topological vector spaces, Math. Ann. 177 (1968), 283-301.

7. R. Brown, The Lefschetz fixed point theorem, Scott, Foresman \& Co., Glenview, Ill., 1971.

8. J. Dugundji and A. Granas, KKM maps and variational inequalities, Ann. Scuola Norm. Sup. Pisa Cl. Sci. 5 (1978), 679-682.

9. K. Fan, Extensions of two fixed point theorems of F. E. Browder, Math. Z. 112 (1969), 234-240.

10. A minimax inequality and applications, Inequalities. III (O. Shisha ed.), Academic Press, New York, 1972, pp. 103-113.

11. J. McClendon, Fixed points of subopen multifunctions, Proc. Amer. Math. Soc. 84 (1982), 425-428.

12.

13. On non-contractibel valued multifunctions, submitted. Subopen multifunctions and selections, Fund. Math. (to appear).

14. R. Thompson, Fixed point indices in locally convex spaces, Adv. in Math. 14 (1974), 73-91.

Department of Mathematics, University of Kansas, LaWrence, Kansas 66045 